\title{
SOME RESULTS CONNECTED WITH A PROBLEM OF ERDÖS. III
}

\author{
J. ARIAS DE REYNA
}

\begin{abstract}
It is shown that if $E$ is a subset with more than two points of the real line, then there exists a subset $S$ of the unit interval, such that $S$ has outer Lebesgue measure one and $S$ is of the second Baire category and such that $S$ does not contain a subset similar (in the sense of elementary geometry) to $E$. This result is related to a conjecture of P. Erdös.
\end{abstract}

1. Introduction. It is known that if $E$ is a finite subset of $\mathbf{R}$ and $A$ a subset of positive Lebesgue measure, then $A$ contains a subset similar (in the sense of elementary geometry) to $E$.

P. Erdös [1] presented the following conjecture at the problem session of the Fifth Balkan Mathematical Congress (Belgrade, June 24-30, 1974):

Conjecture. Let $E$ be an infinite set of real numbers. Then there exists a set of real numbers $S$ of positive Lebesgue measure which does not contain a set $E^{\prime}$ similar to $E$.

H. I. Miller [3] proved, using the continuum hypothesis, that if $E$ is an uncountable subset of the real line, then there exist subsets $S_{1}$ and $S_{2}$ of the unit interval, such that $S_{1}$ has outer Lebesgue measure one and $S_{2}$ is of the second Baire category and such that neither $S_{1}$ nor $S_{2}$ contains a subset similar to $E$.

Here we prove without the continuum hypothesis the following theorem.

THEOREM. Let $E$ be a subset of $\mathbf{R}$ with more than two points. There exists a subset $S$ of the unit interval such that

1. $S$ has outer Lebesgue measure one.

2. $S$ is of the second Baire category at each of its points.

3. $S$ does not contain a subset similar to $E$.

2. Proof. We may assume that $E$ has three points.

Let $\Omega$ be the first ordinal number of the power of the continuum. Suppose $\left\langle x_{\alpha}\right.$ : $\alpha<\Omega\rangle$ (resp. $\left\langle F_{\alpha}: \alpha<\Omega\right\rangle$ ) is a transfinite sequence whose elements are the points of $[0,1]$ (resp. the perfect subsets of $[0,1]$ ). We shall construct by transfinite induction another sequence $\left\langle y_{\alpha}: \alpha<\Omega\right\rangle$, with real numbers, in the following way.

Suppose that if $\beta<\alpha$ we have chosen $y_{\beta}$. Let $A_{\alpha}$ be the set of $x \in \mathbf{R}$ such that there exists a similarity transformation $u$ with $u(E) \subset\{x\} \cup\left\{y_{\beta}: \beta<\alpha\right\}$. Since the

Received by the editors January 18, 1983.

1980 Mathematics Subject Classification. Primary 28A05, 26A21.

Key words and phrases. Lebesgue measure, Baire category. 
cardinality of the set $A_{\alpha}$ is less than the cardinality of the set $6 \times\left\{y_{\beta}: \beta<\alpha\right\} \times\left\{y_{\beta}\right.$ : $\beta<\alpha\}$ we have $\left|A_{\alpha}\right|<2^{\kappa_{0}}$. We call $y_{\alpha}$ the first point in $\left\langle x_{\gamma}: \gamma<\Omega\right\rangle$ contained in $F_{\alpha} \backslash\left(A_{\alpha} \cup\left\{y_{\beta}: \beta<\alpha\right\}\right)$.

Set $S=\left\{y_{\alpha}: \alpha<\Omega\right\}$. It is clear that $S \subset[0,1]$.

Since $y_{\alpha} \in S \cap F_{\alpha}, S$ intersects every perfect subset of $[0,1]$. It is clear that the outer Lebesgue measure of $S$ is one.

$S$ is of second Baire category at each of its points because every uncountable Borel subset of $\mathbf{R}$ contains a perfect subset [2, p. 427].

Finally, by construction, $S$ does not contain a subset similar to $E$.

\section{REFERENCES}

1. P. Erdös, Problems, Math. Balkanica (Papers presented at the Fifth Balkan Mathematical Congress) 4 (1974), 203-204.

2. K. Kuratowski and A. Mostowski, Set theory, 2nd ed., North-Holland, Amsterdam, 1976.

3. H. I. Miller, Some results connected with a problem of Erdös. II, Proc. Amer. Math. Soc. 75 (1979), 265-268.

Facultad de Matemáticas, Universidad de Sevilla, c/ Tarfia SN., Sevilla-12, Spain 